\title{
Toxic Currency Options in Poland as a Consequence of the 2008 Financial Crisis
}

\author{
Kamil Liberadzki \\ Warsaw School of Economics, Warszawa, Poland
}

\begin{abstract}
The paper is on toxic foreign exchange options problem which occurred in Poland just prior to and after the outbreak of the recent crisis. Especially Polish enterprises were severely stricken by transactions on fx and interest rate derivatives contracted with their banks. Poland was the only EU country which did not precipitate into recession during the financial crisis beginning in 2008. However, the toxic $\mathrm{fx}$ and interest rate derivatives transmitted the shockwaves from global financial markets into Poland. Huge dimensions of losses resulted in conflicts between banks and their customers, who claimed just being cheated by the financial institutions. The article deeply researches into reasons for such developments on Polish fx over-the-counter derivatives market. As a case study, an authentic strategy has been presented. The contract was concluded between the construction company and one of the biggest commercial banks in Poland. Because the case study may be representative for many other cases, the analysis includes exact pricing of option strategy and therefore reveals inequality of the contract. The consequences of non-implementing the MiFID directive in the context of derivatives offering to non-financial customers were also touched in the paper.
\end{abstract}

Keywords: toxic foreign exchange options, MiFID, risk reversal, foreign exchange portfolio hedging, exotic options, barrier options, option strategies

\section{Introduction}

What came to be called "toxic" currency options became quite a topic in public debate in Poland late in 2008 and early in 2009. Banks operating in Poland closed currency option positions with export enterprises effecting sales in the main currencies, i.e., the U.S. dollar and the euro. Businesses concluding such option transactions declared that they did so usually in order to hedge against currency risk. They took on short fx positions by selling (or buying) currency options. To close currency positions, banks did not reach for straightforward (elementary) option transactions. What banks offered to practically all clients were characteristic option strategies, consisting mainly of exotic options. These were mostly zero cost contracts, usually iterative structures (with payout, say, every month for one year). Long positions in options were financed through open short positions in the particular options.

The Lehman Brothers collapse undercut currency exchange rates in Central Eastern European countries. Short option position produced immense losses, reaching at least few billion euro.

\footnotetext{
Kamil Liberadzki, Ph.D., junior professor, Institute of Finance, Warsaw School of Economics, Warsaw, Poland.

Correspondence concerning this article should be addressed to Kamil Liberadzki, Instytut Finansów Szkoły Głównej Handlowej, Madalińskiego 6/8 Street, 02-513 Warszawa, Poland.
} 
The main goal of the article is to give an insight into these "toxic" transactions. What was their structure, were they fairly priced? Would be the losses equally high, if instead of shortening fx positions with options, another derivatives (forwards) were used? Finally, the impact of financial regulations on the issue is concerned.

\section{Toxic Derivatives Issue}

\section{Toxic Derivatives as a Cause of Financial Crisis in the Emerging Economies}

Poland was the only EU country which did not precipitate into recession during the financial crisis beginning in 2008. The country sustained its macroeconomic stability; the banking sector remained profitable with high quality credit portfolios (although many loans were denominated in foreign currencies). Apparently, some shockwaves reached Poland as well: Banks granted loans more reluctantly; similarly to other East European currencies, zloty depreciated significantly. There was, however, the one significant channel transmitting contagious shocks from foreign currency market into Polish real economy: namely foreign exchange currency options.

There are numerous researches on causes and impact of different crises of the 1990s and 2000s on emerging economies. Razin and Rosefielde (2011) surveyed three distinct types of crises: banking implosion (Japan); foreign reserves meltdown triggered by hot money flight from economies with fixed exchange rates (developing Asian countries); and finally the 2008 crisis caused by macroeconomic imbalances, spreading across the globe through international financial networks. Cloke (2009) agreed that the US became an epicenter of the last financial crises due to an illusionary market in the increasingly complex credit derivatives. Sikorski (2011) also traced down origins of the crisis to credit securitization and credit derivatives which unleashed a series of major financial institution bankruptcies. Wide spreading credit crunch hit even sound and viable enterprises worldwide.

According to Mullineux (2010), prudential supervision and better regulation of financial innovation in wholesale and investment banking referring to credit derivatives should impose "stress-testing" of financial innovation before they are allowed to be offered to bank customers.

Glass (2009) explored regulatory deficiencies with respect to unsatisfactory collateralization of OTC credit derivatives and lack of central counterparty clearing institutions.

To sum up, researchers identified that the break-down of complex credit derivatives market in the US turned out to be contagious for emerging economies mainly through financial institution insolvencies channel and dysfunction of credit supply. Polish foreign exchange option issue was of quite different kind: The phenomenon was limited to domestic market and option payoff triggered by depreciation of local currency just prior to and after Lehman collapse.

\section{Toxic Foreign Exchange Options in Poland}

The actual amount of losses Polish businesses sustained in currency options trading at the turn of 2008 to 2009 has never been clearly determined. The Polish Financial Supervision Authority (KNF), however, defined the number of businesses involved in currency option operations at several hundred, their aggregate loss at an estimated dozen billion złotys (PLN) or so (Table 1). Alternative evaluations of losses Polish firms suffered as a result of that currency options trading put the estimates at between a dozen and even 200 billion currency! Poland's Central Statistical Office (GUS, 2010) put the aggregate result on 2009 options operations at an estimated PLN 90 billion. The fx rate EUR/PLN at the turn of 2008 to 2009 was more or less 4 (1 EUR $=4$ PLN). 
Some countrywide well-known enterprises suffering losses on such transactions included engineering manufacturer ELWO S.A., foundry corporation Odlewnie Polskie, glassworks Huty Szkła of Krosno (PLN 37.8 million loss), or roof support supplier FAZOS as part of Famur group (PLN 51.8 million). Cersanit, Paged, Sanwil, Forte, and Feroco are more companies to mention in this connection. The last-named company, a trackway supplier, posted a loss to the tune of PLN 160 million. Companies in the PAGED group (furniture and wood products maker) suffered losses amounting to PLN 53 million. The loss-taking firms belonged to various sectors, such as furniture, materials production, building, and assembly services, to mention but a few. It should be noted that to a medium-sized Polish enterprise (with yearly turnover in the range of EUR 50 million), payment of cost in the range of EUR 5-10 million euro was often tantamount to bankruptcy.

Table 1

Estimated Toxic FX Derivative Losses to Businesses (PLN* Billion), KNF Estimates

\begin{tabular}{llllll}
\hline Release date & Updated on & FX option & FX forward & CIRS & Total \\
\hline 17 Dec. 2008 & n.d. & 5.5 & n.d. & n.d. & n.d. \\
11 Mar. 2009 & 13 Feb. 2009 & 9.0 & 7.0 & 2.0 & 18.0 \\
28 Apr. 2009 & 17 Apr. 2009 & 4.5 & 3.6 & 1.1 & 9.2 \\
17 Aug. 2009 & 31 July 2009 & 2.2 & 2.2 & 1.1 & 5.5 \\
\hline
\end{tabular}

Note. *Assume the EUR/PLN exchange rate: 1 EUR $=4$ PLN at the turn of 2008 to 2009. Source: KNF (2008; 2009a; 2009b; 2009c) and Konopczak, Mielus, and Wieprzowski (2011).

The option strategies applied were basically portfolios comprised of vanilla options as well as exotic (barrier or binary) options. These were essentially zero cost strategies, which necessarily involved the simultaneous closure of opposite positions (short and long) in various options, so the premiums of those derivatives must be equal. No reliable estimates of the structure of option portfolios are available, so there is no saying for sure what proportion of such portfolios involved risk reversal strategies (see chapter 2) composed of vanilla options and what strategies comprised of exotic options.

National Bank of Poland-NBP (2009) estimates indicated that currency options made out to the banking sector had dropped in nominal terms, adjusted for exchange rate volatility, approximately $40 \%$ by March 2009 , from September 2009, following settlements of options on maturity, early closures of positions by businesses, or substitution of loans for obligations on options. In certain cases, banks resolved to write off parts of obligations clients had taken out on the currency options contracts (NBP, 2009).

In March 2009, the NBP polled businesses on their experiences in their use of currency options. Of all businesses that answered the (NBP, 2009) questionnaire, 6.7\% declared to have taken advantage of currency options and 3.3\% supplied details of transactions they concluded on the currency options market. Based on those figures, the NBP gave their estimates of how the risk factors implicit in currency options valuation impacted liabilities of the businesses (Table 2).

The figures in Table 2 show that had depreciation of the Polish currency against foreign currencies continued beyond Q 1 2009, Polish businesses would have suffered worse losses.

\section{The Option Strategies Involved}

As of May 2004, Poland has been a member country of the European Union. To Polish businesses, that fact meant a steep rise in foreign trade balances. Early in Q 4 2008, the Polish currency started to gain steadily against the other currencies, which tended to hamper exports (Figure 1). That in turn drove up demand for derivative instruments, mostly on a desire to hedge against the złoty strengthening against foreign currencies. 
That tendency held on for a fairly long time, which prompted businesses to take short positions on currency. The Lehman Brothers collapse dragged down the Polish currency and implied volatility shoot up vehemently (by up to five times the previous level). Most businesses were on short positions on the derivatives market, so vehement a trend reversal along with a rise of implied volatility at the same time was bound to bring huge losses to them following option settlement transactions and adverse valuations of client positions with banks. Many banks therefore pushed clients to reinforce their hedge positions or persuaded them to close old positions (at a loss) and open new option transactions.

Table 2

Impact of Risk Factors Implicit in Currency Options Valuation on Liabilities Balances of the Businesses Polled

\begin{tabular}{lll}
\hline Change of risk factor & Impact on pricing of NBP portfolio (PLN) & $\begin{array}{l}\text { Estimated impact on pricing of total sold } \\
\text { fx option portfolio (PLN) }\end{array}$ \\
\hline $\begin{array}{l}\text { EUR/PLN depreciates PLN } 0.01 \\
\text { (portfolio delta) }\end{array}$ & $-1,956 \mathrm{k}$ & -49 million \\
$\begin{array}{l}\text { Implied volatility rises 1 pp } \\
\text { (vega) }\end{array}$ & $-552 \mathrm{k}$ & -14 million \\
Domestic interest rate cut by 1 pp (rho) & $-34 \mathrm{k}$ & -0.85 million \\
\hline
\end{tabular}

Source: NBP (2009).

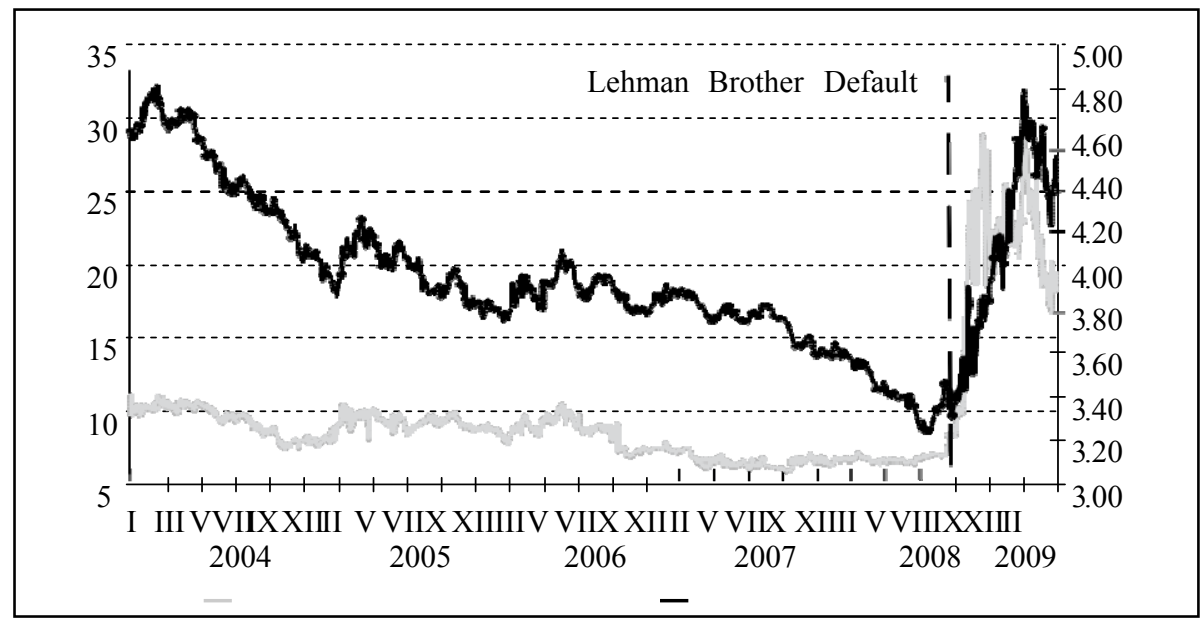

Figure 1. EUR/PLN exchange rate and volatility change 2004-2009. Notes. Market volatility (left axis-light line); EUR/PLN rate (right axis_-bold line). Source: Konopczak et al. (2011).

There are no official or published data investigating into detailed types of these option transactions. According to the research and case studies analysis, the authors can outline three types of the option strategies involved:

- Type I: risk reversals consisting of vanilla option series expiring consecutively;

- Type II: zero-cost portfolios of barrier and/or binary options. They are sometimes called accumulators (Liberadzki, 2014). An accumulator is a type of a barrier option which is typically used in stock markets (T. Kwong, D. Fok, K. K. Kwong, L. Fok, \& W. Fok, 2011). Accumulators are instruments with a double barrier, i.e., an "out" barrier set above the spot price of the underlying instrument and an "in" barrier set below the target price of the underlying asset; in this case, the buyer of the accumulator should purchase double the amount of the underlying instrument. There are many types of accumulators: Vanilla accumulators do not involve a double barrier and geared accumulators involve a double or even triple barrier. These portfolios of exotic options were nicknamed "I will kill you later" in the Southern Asia countries; 
- Type III: Target Accrual Redemption Note (TARN) is a structure including long- and short-option position (thus generating both positive payoffs and negative payouts). There is a cap value on the positive payoffs of the underlying structure.

\section{A "Toxic" Accumulator Strategy Concluded Between a Medium-sized Enterprise and a Major Commercial Bank in Poland-A Case Study}

The real case study presented involves a medium-sized transportation company (the firm) operating in Poland and within the European Union, which closed an option deal with one of major banks in Poland (the bank). This is going to be an example representative for the second type of option strategies, as classified above (in the section 2 of the article), namely combining long down and out barrier put options with short down and out barrier calls.

The selected transactions were concluded on 22th July, 2008. The firm bought 26 put options with the strike price at 3.29 and an American down and out barrier set at 3.13. The notional of each put option was 75,000 euro, the instruments expired consecutively every second week, beginning from the 23th July, 2008 (see Table 3).

Table 3

Pricing the Transaction as on the 22th July 2008

\begin{tabular}{|c|c|c|c|c|c|c|c|c|c|c|}
\hline Spot & 3.223 & & & & & & & & & \\
\hline $\begin{array}{l}\text { Date of signing the } \\
\text { agreement }\end{array}$ & Currency & $\begin{array}{l}\text { Notional } \\
\text { (EUR) }\end{array}$ & Strike & Expiry date & $\begin{array}{l}\text { Barrier down } \\
\text { and out }\end{array}$ & $\begin{array}{l}\text { The firm I } \\
\text { buys/sells }\end{array}$ & Call/put & $\begin{array}{l}\text { Premium } \\
\text { (PLN) }\end{array}$ & $\begin{array}{l}\text { Price in pips } \\
(\text { notional EUR } \times \text { price in } \\
\text { pips }=\text { price in PLN) }\end{array}$ & $\begin{array}{l}\text { Reference } \\
\text { exchange rate }\end{array}$ \\
\hline Tue., 22 July, 2008 & EUR/PLN & 75,000 & 3.29 & Wed., 23 July, 2008 & 3.1300 & Buy & EUR put & $-5,002$ & 0.06669 & 3.2603 \\
\hline Tue., 22 July, 2008 & EUR/PLN & 75,000 & 3.29 & Wed., 6 Aug., 2008 & 3.1300 & Buy & EUR put & $-4,335$ & 0.057805 & 3.2305 \\
\hline Tue., 22 July, 2008 & EUR/PLN & 75,000 & 3.29 & Wed., 20 Aug., 2008 & 3.1300 & Buy & EUR put & $-3,605$ & 0.048072 & 3.3083 \\
\hline Tue., 22 July, 2008 & EUR/PLN & 75,000 & 3.29 & Wed., 3 Sep., 2008 & 3.1300 & Buy & EUR put & $-3,052$ & 0.040687 & 3.3670 \\
\hline Tue., 22 July, 2008 & EUR/PLN & 75,000 & 3.29 & Wed., 17 Sep., 2008 & 3.1300 & Buy & EUR put & $-2,610$ & 0.034798 & 3.3363 \\
\hline Tue., 22 July, 2008 & EUR/PLN & 75,000 & 3.29 & Wed., 1 Oct., 2008 & 3.1300 & Buy & EUR put & $-2,276$ & 0.030346 & 3.3775 \\
\hline Tue., 22 July, 2008 & EUR/PLN & 75,000 & 3.29 & Wed., 15 Oct., 2008 & 3.1300 & Buy & EUR put & $-2,019$ & 0.026923 & 3.5304 \\
\hline Tue., 22 July, 2008 & EUR/PLN & 75,000 & 3.29 & Wed., 29 Oct., 2008 & 3.1300 & Buy & EUR put & $-1,796$ & 0.023943 & 3.6320 \\
\hline Tue., 22 July, 2008 & EUR/PLN & 75,000 & 3.29 & Wed., 12 Nov., 2008 & 3.1300 & Buy & EUR put & $-1,604$ & 0.021391 & 3.7693 \\
\hline Tue., 22 July, 2008 & EUR/PLN & 75,000 & 3.29 & Wed., 26 Nov., 2008 & 3.1300 & Buy & EUR put & $-1,451$ & 0.019343 & 3.7888 \\
\hline Tue., 22 July, 2008 & EUR/PLN & 75,000 & 3.29 & Wed., 10 Dec., 2008 & 3.1300 & Buy & EUR put & $-1,325$ & 0.017668 & 3.9589 \\
\hline Tue., 22 July, 2008 & EUR/PLN & 75,000 & 3.29 & Wed., 24 Dec., 2008 & 3.1300 & Buy & EUR put & $-1,216$ & 0.01621 & 4.1025 \\
\hline Tue., 22 July, 2008 & EUR/PLN & 75,000 & 3.29 & Wed., 7 Jan., 2009 & 3.1300 & Buy & EUR put & $-1,136$ & 0.015144 & 3.9170 \\
\hline Tue., 22 July, 2008 & EUR/PLN & 75,000 & 3.29 & Wed., 21 Jan., 2009 & 3.1300 & Buy & EUR put & $-1,062$ & 0.014158 & 4.3305 \\
\hline Tue., 22 July, 2008 & EUR/PLN & 75,000 & 3.29 & Wed., 4 Feb., 2009 & 3.1300 & Buy & EUR put & -997 & 0.013289 & 4.6747 \\
\hline Tue., 22 July, 2008 & EUR/PLN & 75,000 & 3.29 & Wed., 18 Feb., 2009 & 3.1300 & Buy & EUR put & -927 & 0.012354 & 4.8999 \\
\hline Tue., 22 July, 2008 & EUR/PLN & 75,000 & 3.29 & Wed., 4 Mar., 2009 & 3.1300 & Buy & EUR put & -863 & 0.011506 & 4.7432 \\
\hline Tue., 22 July, 2008 & EUR/PLN & 75,000 & 3.29 & Wed., 18 Mar., 2009 & 3.1300 & Buy & EUR put & -806 & 0.010749 & 4.5313 \\
\hline Tue., 22 July, 2008 & EUR/PLN & 75,000 & 3.29 & Wed., 1 Apr., 2009 & 3.1300 & Buy & EUR put & -751 & 0.010016 & 4.6462 \\
\hline Tue., 22 July, 2008 & EUR/PLN & 75,000 & 3.29 & Wed., 15 Apr., 2009 & 3.1300 & Buy & EUR put & -706 & 0.009419 & 4.2351 \\
\hline Tue., 22 July, 2008 & EUR/PLN & 75,000 & 3.29 & Wed., 29 Apr., 2009 & 3.1300 & Buy & EUR put & -663 & 0.008843 & 4.4498 \\
\hline Tue., 22 July, 2008 & EUR/PLN & 75,000 & 3.29 & Wed., 13 May, 2009 & 3.1300 & Buy & EUR put & -624 & 0.008325 & 4.3943 \\
\hline Tue., 22 July, 2008 & EUR/PLN & 75,000 & 3.29 & Wed., 27 May, 2009 & 3.1300 & Buy & EUR put & -588 & 0.007846 & 4.4313 \\
\hline Tue., 22 July, 2008 & EUR/PLN & 75,000 & 3.29 & Wed., 10 June, 2009 & 3.1300 & Buy & EUR put & -555 & 0.007396 & 4.4729 \\
\hline Tue., 22 July,2008 & EUR/PLN & 75,000 & 3.29 & Wed., 24 June, 2009 & 3.1300 & Buy & EUR put & -526 & 0.007011 & 4.5476 \\
\hline \multirow[t]{2}{*}{ Tue., 22 July, 2008} & EUR/PLN & 75,000 & 3.29 & Wed., 8 July, 2009 & 3.1300 & Buy & EUR put & -498 & 0.006644 & 4.4241 \\
\hline & & & & & & & PUT value & $-40,993$ & & \\
\hline
\end{tabular}


Table 3 continued

\begin{tabular}{|c|c|c|c|c|c|c|c|c|c|c|}
\hline Spot & 3.223 & & & & & & & & & \\
\hline $\begin{array}{l}\text { Date of signing the } \\
\text { agreement }\end{array}$ & Currency & $\begin{array}{l}\text { Notional } \\
\text { (EUR) }\end{array}$ & Strike & Expiry date & $\begin{array}{l}\text { Barrier down } \\
\text { and out }\end{array}$ & $\begin{array}{l}\text { The firm I } \\
\text { buys/sells }\end{array}$ & Call/put & $\begin{array}{l}\text { Premium } \\
\text { (PLN) }\end{array}$ & $\begin{array}{l}\text { Price in pips } \\
(\text { notional EUR } \times \text { price in } \\
\text { pips }=\text { price in PLN) }\end{array}$ & $\begin{array}{l}\text { Reference } \\
\text { exchange rate }\end{array}$ \\
\hline Tue., 22 July, 2008 & EUR/PLN & 150,000 & 3.29 & Wed., 23 July., 2008 & 3.1300 & Sell & EUR call & 0 & 0 & 3.2603 \\
\hline Tue., 22 July, 2008 & EUR/PLN & 150,000 & 3.29 & Wed., 6 Aug., 2008 & 3.1300 & Sell & EUR call & 6 & 0.000043 & 3.2305 \\
\hline Tue., 22 July, 2008 & EUR/PLN & 150,000 & 3.29 & Wed., 20 Aug., 2008 & 3.1300 & Sell & EUR call & 595 & 0.003967 & 3.3083 \\
\hline Tue., 22 July, 2008 & EUR/PLN & 150,000 & 3.29 & Wed., 3 Sep., 2008 & 3.1300 & Sell & EUR call & 1,182 & 0.007878 & 3.3670 \\
\hline Tue., 22 July, 2008 & EUR/PLN & 150,000 & 3.29 & Wed., 17 Sep., 2008 & 3.1300 & Sell & EUR call & 1,761 & 0.011739 & 3.3363 \\
\hline Tue., 22 July, 2008 & EUR/PLN & 150,000 & 3.29 & Wed., 1 Oct., 2008 & 3.1300 & Sell & EUR call & 2,282 & 0.01521 & 3.3775 \\
\hline Tue., 22 July, 2008 & EUR/PLN & 150,000 & 3.29 & Wed., 15 Oct., 2008 & 3.1300 & Sell & EUR call & 2,750 & 0.018334 & 3.5304 \\
\hline Tue., 22 July, 2008 & EUR/PLN & 150,000 & 3.29 & Wed., 29 Oct., 2008 & 3.1300 & Sell & EUR call & 3,190 & 0.021268 & 3.6320 \\
\hline Tue., 22 July, 2008 & EUR/PLN & 150,000 & 3.29 & Wed., 12 Nov., 2008 & 3.1300 & Sell & EUR call & 3,541 & 0.023607 & 3.7693 \\
\hline Tue., 22 July, 2008 & EUR/PLN & 150,000 & 3.29 & Wed., 26 Nov., 2008 & 3.1300 & Sell & EUR call & 3,864 & 0.02576 & 3.7888 \\
\hline Tue., 22 July, 2008 & EUR/PLN & 150,000 & 3.29 & Wed., 10 Dec., 2008 & 3.1300 & Sell & EUR call & 4,166 & 0.027771 & 3.9589 \\
\hline Tue., 22 July, 2008 & EUR/PLN & 150,000 & 3.29 & Wed., 24 Dec., 2008 & 3.1300 & Sell & EUR call & 4,456 & 0.029707 & 4.1025 \\
\hline Tue., 22 July, 2008 & EUR/PLN & 150,000 & 3.29 & Wed., 7 Jan., 2009 & 3.1300 & Sell & EUR call & 4,739 & 0.031592 & 3.9170 \\
\hline Tue., 22 July, 2008 & EUR/PLN & 150,000 & 3.29 & Wed., 21 Jan., 2009 & 3.1300 & Sell & EUR call & 4,993 & 0.033287 & 4.3305 \\
\hline Tue., 22 July, 2008 & EUR/PLN & 150,000 & 3.29 & Wed., 4 Feb., 2009 & 3.1300 & Sell & EUR call & 5,204 & 0.034695 & 4.6747 \\
\hline Tue., 22 July, 2008 & EUR/PLN & 150,000 & 3.29 & Wed., 18 Feb., 2009 & 3.1300 & Sell & EUR call & 5,402 & 0.03601 & 4.8999 \\
\hline Tue., 22 July, 2008 & EUR/PLN & 150,000 & 3.29 & Wed., 4 Mar., 2009 & 3.1300 & Sell & EUR call & 5,594 & 0.03729 & 4.7432 \\
\hline Tue., 22 July, 2008 & EUR/PLN & 150,000 & 3.29 & Wed., 18 Mar., 2009 & 3.1300 & Sell & EUR call & 5,778 & 0.038517 & 4.5313 \\
\hline Tue., 22 July, 2008 & EUR/PLN & 150,000 & 3.29 & Wed., 1 Apr., 2009 & 3.1300 & Sell & EUR call & 5,955 & 0.039703 & 4.6462 \\
\hline Tue., 22 July, 2008 & EUR/PLN & 150,000 & 3.29 & Wed., 15 Apr., 2009 & 3.1300 & Sell & EUR call & 6,125 & 0.040832 & 4.2351 \\
\hline Tue., 22 July, 2008 & EUR/PLN & 150,000 & 3.29 & Wed., 29 Apr., 2009 & 3.1300 & Sell & EUR call & 6,294 & 0.041958 & 4.4498 \\
\hline Tue., 22 July, 2008 & EUR/PLN & 150,000 & 3.29 & Wed., 13 May, 2009 & 3.1300 & Sell & EUR call & 6,459 & 0.043058 & 4.3943 \\
\hline Tue., 22 July, 2008 & EUR/PLN & 150,000 & 3.29 & Wed., 27 May, 2009 & 3.1300 & Sell & EUR call & 6,623 & 0.044152 & 4.4313 \\
\hline Tue., 22 July, 2008 & EUR/PLN & 150,000 & 3.29 & Wed., 10 June, 2009 & 3.1300 & Sell & EUR call & 6,792 & 0.045278 & 4.4729 \\
\hline Tue., 22 July, 2008 & EUR/PLN & 150,000 & 3.29 & Wed., 24 June, 2009 & 3.1300 & Sell & EUR call & 6,940 & 0.046264 & 4.5476 \\
\hline \multirow[t]{2}{*}{ Tue., 22 July, 2008} & EUR/PLN & 150,000 & 3.29 & Wed., 8 July, 2009 & 3.1300 & Sell & EUR call & 7,095 & 0.047301 & 4.4241 \\
\hline & & & & & & & Call value & $111,783.15$ & & \\
\hline
\end{tabular}

Source: Own study.

Simultaneously, the bank bought 26 call options with an American down and out barrier set at 3.13 written by the firm. Nominal value of each consecutively expiring option was 150,000 euro. The first strike date was set on the 23th July, 2008, the last option expired on the 8th July, 2009 (details of the transaction presented in the Table 3). It shows which options were exercised and what was the payoff. In total, the "firm I" occurred losses equal to $1,742,280$ PLN as a result of exercising short calls. The barrier has never been hit, so the structure was active throughout the period of Polish currency sudden depreciation (see the section 2).

Table 3 contains pricing therefore reveals the profit margin of the "bank I" assuming contemporary foreign exchange market quotation. DeRosa (2011) gave a comprehensive description of models used for different $\mathrm{fx}$ option types pricing. As far as this analysis is concerned, it is crucial to apply a model consistent with those used by the industry at the time the option transactions were concluded. The Black-Scholes-Merton (BSM) approach is a standard pricing method used for the vanilla options directly and forms a basis for exotic option pricing formulas. The market implied volatility surfaces are quoted just being calculated by inverting the BSM pricing equation. 
These volatilities become an input for pricing all strategies with reference to contemporary market quotations. When it comes to European barrier option pricing, following formula will be applied (Haug, 2007). Referring to American options or exotic structures like TARN, appropriate Monte Carlo technique will be used.

Going back to pricing results, the firm "overpaid" for the long put option approx. 40,000 PLN. What is more, the firm valued sold call option at approx. PLN 111,000 less than their actual value. To sum up, if it was not for bank margin, the firm should have received an upfront cash flow of approx. 40,000 $+111,000=$ 150,000 PLN as a fair remuneration for this theoretically "zero cost" option strategy.

Assuming that authors get rid of this bank margin by either improving the nominal of put option or reducing nominal value of short call option in such a way, the price of long puts and short calls equals. Table 4 shows one of possible solutions: The long call notional has been reduced (from 150,000 euro for each option to just 60,000 euro). It resulted in drop of mismatch between calls and puts value from ca. PLN 150,000 to PLN 4,000 .

\section{Table 4}

Pricing the Modified Transaction of 22th July 2008, Excluding the Bank I Margin by Reducing Call Options Nominal

\begin{tabular}{|c|c|c|c|c|c|c|c|c|c|}
\hline Spot & 3,223 & & & & & & & & \\
\hline $\begin{array}{l}\text { Date of signing the } \\
\text { agreement }\end{array}$ & Currency & $\begin{array}{l}\text { Notional } \\
\text { EUR }\end{array}$ & Strike & Expiry & $\begin{array}{l}\text { Barrier down } \\
\& \text { out }\end{array}$ & $\begin{array}{l}\text { The bank I } \\
\text { buys/sells }\end{array}$ & Call/put & Price (PLN) & $\begin{array}{l}\text { Price in pips (notional EUR } \times \\
\text { price in pips = price in PLN) }\end{array}$ \\
\hline Tue., 22 July, 2008 & EUR/PLN & 75,000 & 3.29 & Wed., 23 July, 2008 & 3.13 & Buy & EUR put & $5,001.75$ & 0.06669 \\
\hline Tue., 22 July, 2008 & EUR/PLN & 75,000 & 3.29 & Wed., 6 Aug., 2008 & 3.13 & Buy & EUR put & $4,335.375$ & 0.057805 \\
\hline Tue., 22 July, 2008 & EUR/PLN & 75,000 & 3.29 & Wed., 20 Aug., 2008 & 3.13 & Buy & EUR put & $3,605.4$ & 0.048072 \\
\hline Tue., 22 July, 2008 & EUR/PLN & 75,000 & 3.29 & Wed., 3 Sep., 2008 & 3.13 & Buy & EUR put & $3,051.525$ & 0.040687 \\
\hline Tue., 22 July, 2008 & EUR/PLN & 75,000 & 3.29 & Wed., 17 Sep., 2008 & 3.13 & Buy & EUR put & $2,609.85$ & 0.034798 \\
\hline Tue., 22 July, 2008 & EUR/PLN & 75,000 & 3.29 & Wed., 1 Oct., 2008 & 3.13 & Buy & EUR put & $2,275.95$ & 0.030346 \\
\hline Tue., 22 July, 2008 & EUR/PLN & 75,000 & 3.29 & Wed., 15 Oct., 2008 & 3.13 & Buy & EUR put & $2,019.225$ & 0.026923 \\
\hline Tue., 22 July, 2008 & EUR/PLN & 75,000 & 3.29 & Wed., 29 Oct., 2008 & 3.13 & Buy & EUR put & $1,795.725$ & 0.023943 \\
\hline Tue., 22 July, 2008 & EUR/PLN & 75,000 & 3.29 & Wed., 12 Nov., 2008 & 3.13 & Buy & EUR put & $1,604.325$ & 0.021391 \\
\hline Tue., 22 July, 2008 & EUR/PLN & 75,000 & 3.29 & Wed., 26 Nov., 2008 & 3.13 & Buy & EUR put & $1,450.725$ & 0.019343 \\
\hline Tue., 22 July, 2008 & EUR/PLN & 75,000 & 3.29 & Wed., 10 Dec., 2008 & 3.13 & Buy & EUR put & $1,325.1$ & 0.017668 \\
\hline Tue., 22 July, 2008 & EUR/PLN & 75,000 & 3.29 & Wed., 24 Dec., 2008 & 3.13 & Buy & EUR put & $1,215.75$ & 0.01621 \\
\hline Tue., 22 July, 2008 & EUR/PLN & 75,000 & 3.29 & Wed., 7 Jan., 2009 & 3.13 & Buy & EUR put & $1,135.8$ & 0.015144 \\
\hline Tue., 22 July, 2008 & EUR/PLN & 75,000 & 3.29 & Wed., 21 Jan., 2009 & 3.13 & Buy & EUR put & $1,061.85$ & 0.014158 \\
\hline Tue., 22 July, 2008 & EUR/PLN & 75,000 & 3.29 & Wed., 4 Feb., 2009 & 3.13 & Buy & EUR put & 996.675 & 0.013289 \\
\hline Tue., 22 July, 2008 & EUR/PLN & 75,000 & 3.29 & Wed., 18 Feb., 2009 & 3.13 & Buy & EUR put & 926.55 & 0.012354 \\
\hline Tue., 22 July, 2008 & EUR/PLN & 75,000 & 3.29 & Wed., 4 Mar., 2009 & 3.13 & Buy & EUR put & 862.95 & 0.011506 \\
\hline Tue., 22 July, 2008 & EUR/PLN & 75,000 & 3.29 & Wed., 18 Mar., 2009 & 3.13 & Buy & EUR put & 806.175 & 0.010749 \\
\hline Tue., 22 July, 2008 & EUR/PLN & 75,000 & 3.29 & Wed., 1 Apr., 2009 & 3.13 & Buy & EUR put & 751.2 & 0.010016 \\
\hline Tue., 22 July, 2008 & EUR/PLN & 75,000 & 3.29 & Wed., 15 Apr., 2009 & 3.13 & Buy & EUR put & 706.425 & 0.009419 \\
\hline Tue., 22 July, 2008 & EUR/PLN & 75,000 & 3.29 & Wed., 29 Apr., 2009 & 3.13 & Buy & EUR put & 663.225 & 0.008843 \\
\hline Tue., 22 July, 2008 & EUR/PLN & 75,000 & 3.29 & Wed., 13 May, 2009 & 3.13 & Buy & EUR put & 624.375 & 0.008325 \\
\hline Tue., 22 July, 2008 & EUR/PLN & 75,000 & 3.29 & Wed., 27 May, 2009 & 3.13 & Buy & EUR put & 588.45 & 0.007846 \\
\hline Tue., 22 July, 2008 & EUR/PLN & 75,000 & 3.29 & Wed., 10 June, 2009 & 3.13 & Buy & EUR put & 554.7 & 0.007396 \\
\hline Tue., 22 July, 2008 & EUR/PLN & 75,000 & 3.29 & Wed., 24 June, 2009 & 3.13 & Buy & EUR put & 525.825 & 0.007011 \\
\hline \multirow[t]{2}{*}{ Tue., 22 July, 2008} & EUR/PLN & 75,000 & 3.29 & Wed., 8 July, 2009 & 3.13 & Buy & EUR put & 498.3 & 0.006644 \\
\hline & & & & & & & Total & $40,993.2$ & \\
\hline
\end{tabular}


Table 4 continued

\begin{tabular}{|c|c|c|c|c|c|c|c|c|c|}
\hline $\begin{array}{l}\text { Date of signing the } \\
\text { agreement }\end{array}$ & Currency & $\begin{array}{l}\text { Notional } \\
\text { EUR }\end{array}$ & Strike & Expiry & $\begin{array}{l}\text { Barrier down } \\
\& \text { out }\end{array}$ & $\begin{array}{l}\text { The bank I } \\
\text { buys/sells }\end{array}$ & Call/put & Price (PLN) & $\begin{array}{l}\text { Price in pips (notional EUR } \times \\
\text { price in pips = price in PLN) }\end{array}$ \\
\hline Tue., 22 July, 2008 & EUR/PLN & 60,000 & 3.29 & Wed., 23 July, 2008 & 3.13 & Sell & EUR call & 0 & 0 \\
\hline Tue., 22 July, 2008 & EUR/PLN & 60,000 & 3.29 & Wed., 6 Aug., 2008 & 3.13 & Sell & EUR call & 2.58 & 0.000043 \\
\hline Tue., 22 July, 2008 & EUR/PLN & 60,000 & 3.29 & Wed., 20 Aug., 2008 & 3.13 & Sell & EUR call & 238.02 & 0.003967 \\
\hline Tue., 22 July, 2008 & EUR/PLN & 60,000 & 3.29 & Wed., 3 Sep., 2008 & 3.13 & Sell & EUR call & 472.68 & 0.007878 \\
\hline Tue., 22 July, 2008 & EUR/PLN & 60,000 & 3.29 & Wed., 17 Sep., 2008 & 3.13 & Sell & EUR call & 704.34 & 0.011739 \\
\hline Tue., 22 July, 2008 & EUR/PLN & 60,000 & 3.29 & Wed., 1 Oct., 2008 & 3.13 & Sell & EUR call & 912.6 & 0.01521 \\
\hline Tue., 22 July, 2008 & EUR/PLN & 60,000 & 3.29 & Wed., 15 Oct., 2008 & 3.13 & Sell & EUR call & $1,100.04$ & 0.018334 \\
\hline Tue., 22 July, 2008 & EUR/PLN & 60,000 & 3.29 & Wed., 29 Oct., 2008 & 3.13 & Sell & EUR call & $1,276.08$ & 0.021268 \\
\hline Tue., 22 July, 2008 & EUR/PLN & 60,000 & 3.29 & Wed., 12 Nov., 2008 & 3.13 & Sell & EUR call & $1,416.42$ & 0.023607 \\
\hline Tue., 22 July, 2008 & EUR/PLN & 60,000 & 3.29 & Wed., 26 Nov., 2008 & 3.13 & Sell & EUR call & $1,545.6$ & 0.02576 \\
\hline Tue., 22 July, 2008 & EUR/PLN & 60,000 & 3.29 & Wed., 10 Dec., 2008 & 3.13 & Sell & EUR call & $1,666.26$ & 0.027771 \\
\hline Tue., 22 July, 2008 & EUR/PLN & 60,000 & 3.29 & Wed., 24 Dec., 2008 & 3.13 & Sell & EUR call & $1,782.42$ & 0.029707 \\
\hline Tue., 22 July, 2008 & EUR/PLN & 60,000 & 3.29 & Wed., 7 Jan., 2009 & 3.13 & Sell & EUR call & $1,895.52$ & 0.031592 \\
\hline Tue., 22 July, 2008 & EUR/PLN & 60,000 & 3.29 & Wed., 21 Jan., 2009 & 3.13 & Sell & EUR call & $1,997.22$ & 0.033287 \\
\hline Tue., 22 July, 2008 & EUR/PLN & 60,000 & 3.29 & Wed., 4 Feb., 2009 & 3.13 & Sell & EUR call & $2,081.7$ & 0.034695 \\
\hline Tue., 22 July, 2008 & EUR/PLN & 60,000 & 3.29 & Wed., 18 Feb., 2009 & 3.13 & Sell & EUR call & $2,160.6$ & 0.03601 \\
\hline Tue., 22 July, 2008 & EUR/PLN & 60,000 & 3.29 & Wed., 4 Mar., 2009 & 3.13 & Sell & EUR call & $2,237.4$ & 0.03729 \\
\hline Tue., 22 July, 2008 & EUR/PLN & 60,000 & 3.29 & Wed., 18 Mar., 2009 & 3.13 & Sell & EUR call & $2,311.02$ & 0.038517 \\
\hline Tue., 22 July, 2008 & EUR/PLN & 60,000 & 3.29 & Wed., 1 Apr., 2009 & 3.13 & Sell & EUR call & $2,382.18$ & 0.039703 \\
\hline Tue., 22 July, 2008 & EUR/PLN & 60,000 & 3.29 & Wed., 15 Apr., 2009 & 3.13 & Sell & EUR call & $2,449.92$ & 0.040832 \\
\hline Tue., 22 July, 2008 & EUR/PLN & 60,000 & 3.29 & Wed., 29 Apr., 2009 & 3.13 & Sell & EUR call & $2,517.48$ & 0.041958 \\
\hline Tue., 22 July, 2008 & EUR/PLN & 60,000 & 3.29 & Wed., 13 May, 2009 & 3.13 & Sell & EUR call & $2,583.48$ & 0.043058 \\
\hline Tue., 22 July, 2008 & EUR/PLN & 60,000 & 3.29 & Wed., 27 May, 2009 & 3.13 & Sell & EUR call & $2,649.12$ & 0.044152 \\
\hline Tue., 22 July, 2008 & EUR/PLN & 60,000 & 3.29 & Wed., 10 June, 2009 & 3.13 & Sell & EUR call & $2,716.68$ & 0.045278 \\
\hline Tue., 22 July, 2008 & EUR/PLN & 60,000 & 3.29 & Wed., 24 June, 2009 & 3.13 & Sell & EUR call & $2,775.84$ & 0.046264 \\
\hline \multirow[t]{2}{*}{ Tue., 22 July, 2008} & EUR/PLN & 60,000 & 3.29 & Wed., 8 July, 2009 & 3.13 & Sell & EUR call & $2,838.06$ & 0.047301 \\
\hline & & & & & & & Total & $44,713.26$ & \\
\hline
\end{tabular}

Source: Own study.

Similar outcome may be produced by increasing the puts notional from 75,000 euro for each option to a nominal value of 200,000 euro.

Table 5 shows payoff comparison for real parameters and modified transactions assuming that the "bank I" margin would be transformed into improving transaction parameters as explained above.

In face of an actual decline of zloty's value, only the reduction of short call option notional would diminish the "firm I" losses. According to the calculations (Table 5), zeroing "bank I" margin by call option nominal value reduction would produce a loss on transaction of approx. PLN 693,000 (comparing to PLN 1.7 $\mathrm{m}$ of actual losses). Alternatively reducing the put nominal would yield no gains for the "firm I". So the effectiveness of margin reduction in the simulation depends solely of unpredictable development of future $\mathrm{fx}$ rates. In July 2008, zloty was still following the long-term upsurge trend. 
Table 5

Realized Total Payoff and Simulated Payoff for the Variants With No Margin

\begin{tabular}{|c|c|c|c|c|c|c|c|c|c|c|c|c|c|c|c|c|}
\hline $\begin{array}{l}\text { Option type } \\
\text { BAR = } \\
\text { barrier option }\end{array}$ & $\begin{array}{l}\text { The bank I } \\
\text { buys/sells }\end{array}$ & Call/put & Strike & $\begin{array}{l}\text { Notional } \\
\text { EUR }\end{array}$ & $\begin{array}{l}\text { Notional } \\
\text { PLN }\end{array}$ & Currencies & $\begin{array}{l}\text { Date of } \\
\text { signing the } \\
\text { agreement }\end{array}$ & Expiry date & Settlement date & Barrier & Settlement type & & $\begin{array}{l}\text { Reference } \\
\text { exchange rate }\end{array}$ & $\begin{array}{l}\text { Payoff PLN } \\
\text { ("-"” the bank I } \\
\text { pays; “+”" the } \\
\text { bank I } \\
\text { receives) }\end{array}$ & $\begin{array}{l}\text { Payoff } \\
\text { simulation—call } \\
\text { option nominal } \\
\text { reduced to } \\
60,000 \text { EUR }\end{array}$ & $\begin{array}{l}\text { Payoff } \\
\text { simulation-put } \\
\text { option nominal } \\
\text { reduced to } \\
200,000 \text { EUR } \\
\end{array}$ \\
\hline BAR & Sell & Put & 3.29 & $75,000.00$ & $246,750.00$ & EUR/PLN & $2008-7-22$ & $2008-7-23$ & $2008-7-25$ & 3.13 & Settled & fx settlement & 3.2603 & $-2,227.50$ & $-2,227.50$ & $-5,940.00$ \\
\hline BAR & Sell & Put & 3.29 & $75,000.00$ & $246,750.00$ & EUR/PLN & $2008-7-22$ & $2008-8-6$ & $2008-8-8$ & 3.13 & Settled & fx settlement & 3.2305 & $-4,462.00$ & $-4,462.00$ & $-11,898.67$ \\
\hline BAR & Sell & Put & 3.29 & $75,000.00$ & $246,750.00$ & EUR/PLN & $2008-7-22$ & $2008-8-20$ & $2008-8-22$ & 3.13 & $\begin{array}{l}\text { Expired with no } \\
\text { payoff }\end{array}$ & & 3.3083 & 0.00 & 0.00 & 0.00 \\
\hline BAR & Sell & Put & 3.29 & $75,000.00$ & $246,750.00$ & EUR/PLN & $2008-7-22$ & $2008-9-3$ & 2008-9-5 & 3.13 & $\begin{array}{l}\text { Expired with no } \\
\text { payoff }\end{array}$ & & 3.3670 & 0.00 & 0.00 & 0.00 \\
\hline BAR & Sell & Put & 3.29 & $75,000.00$ & $246,750.00$ & EUR/PLN & $2008-7-22$ & $2008-9-17$ & 2008-9-19 & 3.13 & $\begin{array}{l}\text { Expired with no } \\
\text { payoff }\end{array}$ & - & 3.3363 & 0.00 & 0.00 & 0.00 \\
\hline BAR & Sell & Put & 3.29 & $75,000.00$ & $246,750.00$ & EUR/PLN & $2008-7-22$ & 2008-10-1 & $2008-10-3$ & 3.13 & $\begin{array}{l}\text { Expired with no } \\
\text { payoff }\end{array}$ & - & 3.3775 & 0.00 & 0.00 & 0.00 \\
\hline BAR & Sell & Put & 3.29 & $75,000.00$ & $246,750.00$ & EUR/PLN & $2008-7-22$ & 2008-10-15 & $2008-10-17$ & 3.13 & $\begin{array}{l}\text { Expired with no } \\
\text { payoff }\end{array}$ & & 3.5304 & 0.00 & 0.00 & 0.00 \\
\hline BAR & Sell & Put & 3.29 & $75,000.00$ & $246,750.00$ & EUR/PLN & $2008-7-22$ & 2008-10-29 & 2008-10-31 & 3.13 & $\begin{array}{l}\text { Expired with no } \\
\text { payoff }\end{array}$ & - & 3.6320 & 0.00 & 0.00 & 0.00 \\
\hline BAR & Sell & Put & 3.29 & $75,000.00$ & $246,750.00$ & EUR/PLN & $2008-7-22$ & 2008-11-12 & 2008-11-14 & 3.13 & $\begin{array}{l}\text { Expired with no } \\
\text { payoff }\end{array}$ & - & 3.7693 & 0.00 & 0.00 & 0.00 \\
\hline BAR & Sell & Put & 3.29 & $75,000.00$ & $246,750.00$ & EUR/PLN & $2008-7-22$ & $2008-11-26$ & $2008-11-28$ & 3.13 & $\begin{array}{l}\text { Expired with no } \\
\text { payoff }\end{array}$ & & 3.7888 & 0.00 & 0.00 & 0.00 \\
\hline BAR & Sell & Put & 3.29 & $75,000.00$ & $246,750.00$ & EUR/PLN & $2008-7-22$ & $2008-12-10$ & $2008-12-12$ & 3.13 & $\begin{array}{l}\text { Expired with no } \\
\text { payoff }\end{array}$ & - & 3.9589 & 0.00 & 0.00 & 0.00 \\
\hline BAR & Sell & Put & 3.29 & $75,000.00$ & $246,750.00$ & EUR/PLN & $2008-7-22$ & $2008-12-24$ & $2008-12-30$ & 3.13 & $\begin{array}{l}\text { Expired with no } \\
\text { payoff }\end{array}$ & - & - & - & - & \\
\hline BAR & Sell & Put & 3.29 & $75,000.00$ & $246,750.00$ & EUR/PLN & $2008-7-22$ & $2009-1-7$ & 2009-1-9 & 3.13 & $\begin{array}{l}\text { Expired with no } \\
\text { payoff }\end{array}$ & & 3.9170 & 0.00 & 0.00 & 0.00 \\
\hline BAR & Sell & Put & 3.29 & $75,000.00$ & $246,750.00$ & EUR/PLN & $2008-7-22$ & $2009-1-21$ & $2009-1-23$ & 3.13 & $\begin{array}{l}\text { Expired with no } \\
\text { payoff }\end{array}$ & & 4.3305 & 0.00 & 0.00 & 0.00 \\
\hline BAR & Sell & Put & 3.29 & $75,000.00$ & $246,750.00$ & EUR/PLN & $2008-7-22$ & $2009-2-4$ & 2009-2-6 & 3.13 & $\begin{array}{l}\text { Expired with no } \\
\text { payoff }\end{array}$ & - & 4.6747 & 0.00 & 0.00 & 0.00 \\
\hline BAR & Sell & Put & 3.29 & $75,000.00$ & $246,750.00$ & EUR/PLN & $2008-7-22$ & $2009-2-18$ & $2009-2-20$ & 3.13 & $\begin{array}{l}\text { Expired with no } \\
\text { payoff }\end{array}$ & - & 4.8999 & 0.00 & 0.00 & 0.00 \\
\hline BAR & Sell & Put & 3.29 & $75,000.00$ & $246,750.00$ & EUR/PLN & $2008-7-22$ & $2009-3-4$ & 2009-3-6 & 3.13 & $\begin{array}{l}\text { Expired with no } \\
\text { payoff }\end{array}$ & & 4.7432 & 0.00 & 0.00 & 0.00 \\
\hline BAR & Sell & Put & 3.29 & $75,000.00$ & $246,750.00$ & EUR/PLN & $2008-7-22$ & 2009-3-18 & $2009-3-20$ & 3.13 & $\begin{array}{l}\text { Expired with no } \\
\text { payoff }\end{array}$ & - & 4.5313 & 0.00 & 0.00 & 0.00 \\
\hline BAR & Sell & Put & 3.29 & $75,000.00$ & $246,750.00$ & EUR/PLN & $2008-7-22$ & 2009-4-1 & 2009-4-3 & 3.13 & $\begin{array}{l}\text { Expired with no } \\
\text { payoff }\end{array}$ & - & 4.6462 & 0.00 & 0.00 & 0.00 \\
\hline BAR & Sell & Put & 3.29 & $75,000.00$ & $246,750.00$ & EUR/PLN & $2008-7-22$ & 2009-4-15 & 2009-4-17 & 3.13 & $\begin{array}{l}\text { Expired with no } \\
\text { payoff }\end{array}$ & & 4.2351 & 0.00 & 0.00 & 0.00 \\
\hline BAR & Sell & Put & 3.29 & $75,000.00$ & $246,750.00$ & EUR/PLN & $2008-7-22$ & 2009-4-29 & $2009-5-4$ & 3.13 & $\begin{array}{l}\text { Expired with no } \\
\text { payoff }\end{array}$ & - & 4.4498 & 0.00 & 0.00 & 0.00 \\
\hline BAR & Sell & Put & 3.29 & $75,000.00$ & $246,750.00$ & EUR/PLN & 2008-7-22 & 2009-5-13 & 2009-5-15 & 3.13 & $\begin{array}{l}\text { Expired with no } \\
\text { payoff }\end{array}$ & - & 4.3943 & 0.00 & 0.00 & 0.00 \\
\hline BAR & Sell & Put & 3.29 & $75,000.00$ & $246,750.00$ & EUR/PLN & $2008-7-22$ & $2009-5-27$ & 2009-5-29 & 3.13 & $\begin{array}{l}\text { Expired with no } \\
\text { payoff } \\
\text { The option }\end{array}$ & & 4.4313 & 0.00 & 0.00 & 0.00 \\
\hline BAR & Sell & Put & 3.29 & $75,000.00$ & $246,750.00$ & EUR/PLN & $2008-7-22$ & 2009-6-10 & 2009-6-15 & 3.13 & $\begin{array}{l}\text { bought backed } \\
09 / 06 / 09 \\
\text { The option }\end{array}$ & - & - & - & - & \\
\hline BAR & Sell & Put & 3.29 & $75,000.00$ & $246,750.00$ & EUR/PLN & $2008-7-22$ & $2009-6-24$ & 2009-6-26 & 3.13 & $\begin{array}{l}\text { bought backed } \\
09 / 06 / 10\end{array}$ & & - & - & - & \\
\hline
\end{tabular}




\begin{tabular}{|c|c|c|c|c|c|c|c|c|c|c|c|c|c|c|c|c|}
\hline $\begin{array}{l}\text { Option type } \\
\text { BAR = } \\
\text { barrier option }\end{array}$ & $\begin{array}{l}\text { The bank I } \\
\text { buys/sells }\end{array}$ & Call/put & Strike & $\begin{array}{l}\text { Notional } \\
\text { EUR }\end{array}$ & $\begin{array}{l}\text { Notional } \\
\text { PLN }\end{array}$ & Currencies & $\begin{array}{l}\text { Date of } \\
\text { signing the } \\
\text { agreement }\end{array}$ & Expiry date & Settlement date & Barrier & Settlement type & & $\begin{array}{l}\text { Reference } \\
\text { exchange rate }\end{array}$ & $\begin{array}{l}\text { Payoff PLN } \\
\text { (“-” the bank I } \\
\text { pays; “+”” the } \\
\text { bank I } \\
\text { receives) } \\
\end{array}$ & $\begin{array}{l}\text { Payoff } \\
\text { simulation—call } \\
\text { option nominal } \\
\text { reduced to } \\
60,000 \text { EUR } \\
\end{array}$ & $\begin{array}{l}\text { Payoff } \\
\text { simulation-put } \\
\text { option nominal } \\
\text { reduced to } \\
200,000 \text { EUR } \\
\end{array}$ \\
\hline BAR & Sell & Put & 3.29 & $75,000.00$ & $246,750.00$ & EUR/PLN & $2008-7-22$ & $2009-7-8$ & 2008-7-25 & 3.13 & $\begin{array}{l}\text { The option } \\
\text { bought backed } \\
09 / 06 / 11\end{array}$ & - & - & - & - & \\
\hline BAR & Buy & Call & 3.29 & $150,000.00$ & $493,500.00$ & EUR/PLN & $2008-7-22$ & $2008-7-23$ & $2008-8-8$ & 3.13 & $\begin{array}{l}\text { Expired with no } \\
\text { payoff }\end{array}$ & - & 3.2603 & 0.00 & 0.00 & 0.00 \\
\hline BAR & Buy & Call & 3.29 & $150,000.00$ & $493,500.00$ & EUR/PLN & $2008-7-22$ & $2008-8-6$ & 2008-8-22 & 3.13 & $\begin{array}{l}\text { Expired with no } \\
\text { payoff }\end{array}$ & - & 3.2305 & 0.00 & 0.00 & 0.00 \\
\hline BAR & Buy & Call & 3.29 & $150,000.00$ & $493,500.00$ & EUR/PLN & 2008-7-22 & $2008-8-20$ & $2008-9-5$ & 3.13 & Settled & fx settlement & 3.3083 & $2,745.00$ & $1,098.00$ & $2,745.00$ \\
\hline BAR & Buy & Call & 3.29 & $150,000.00$ & $493,500.00$ & EUR/PLN & 2008-7-22 & $2008-9-3$ & 2008-9-19 & 3.13 & Settled & fx settlement & 3.3670 & $11,550.00$ & $4,620.00$ & $11,550.00$ \\
\hline BAR & Buy & Call & 3.29 & $150,000.00$ & $493,500.00$ & EUR/PLN & $2008-7-22$ & 2008-9-17 & $2008-10-3$ & 3.13 & Settled & fx settlement & 3.3363 & $6,945.00$ & $2,778.00$ & $6,945.00$ \\
\hline BAR & Buy & Call & 3.29 & $150,000.00$ & $493,500.00$ & EUR/PLN & 2008-7-22 & 2008-10-1 & 2008-10-17 & 3.13 & Settled & fx settlement & 3.3775 & $13,125.00$ & $5,250.00$ & $13,125.00$ \\
\hline BAR & Buy & Call & 3.29 & $150,000.00$ & $493,500.00$ & EUR/PLN & $2008-7-22$ & $2008-10-15$ & 2008-10-31 & 3.13 & $\begin{array}{l}\text { Expired with no } \\
\text { payoff }\end{array}$ & - & 3.5304 & 0.00 & 0.00 & 0.00 \\
\hline BAR & Buy & Call & 3.29 & $150,000.00$ & $493,500.00$ & EUR/PLN & 2008-7-22 & $2008-10-29$ & 2008-11-14 & 3.13 & Settled & fx settlement & 3.6320 & $51,300.00$ & $20,520.00$ & $51,300.00$ \\
\hline BAR & Buy & Call & 3.29 & $150,000.00$ & $493,500.00$ & EUR/PLN & $2008-7-22$ & 2008-11-12 & $2008-11-28$ & 3.13 & Settled & fx settlement & 3.7693 & $71,895.00$ & $28,758.00$ & $71,895.00$ \\
\hline BAR & Buy & Call & 3.29 & $150,000.00$ & $493,500.00$ & EUR/PLN & 2008-7-22 & $2008-11-26$ & $2008-12-12$ & 3.13 & Settled & fx settlement & 3.7888 & $74,820.00$ & $29,928.00$ & $74,820.00$ \\
\hline BAR & Buy & Call & 3.29 & $150,000.00$ & $493,500.00$ & EUR/PLN & 2008-7-22 & 2008-12-10 & $2008-12-30$ & 3.13 & Settled & fx settlement & 3.9589 & $100,335.00$ & $40,134.00$ & $100,335.00$ \\
\hline BAR & Buy & Call & 3.29 & $150,000.00$ & $493,500.00$ & EUR/PLN & $2008-7-22$ & 2008-12-24 & 2009-1-9 & 3.13 & $\begin{array}{l}\text { The option sold } \\
\text { back }\end{array}$ & - & - & - & & \\
\hline BAR & Buy & Call & 3.29 & $150,000.00$ & $493,500.00$ & EUR/PLN & $2008-7-22$ & $2009-1-7$ & $2009-1-23$ & 3.13 & $\begin{array}{l}\text { The option sold } \\
\text { back }\end{array}$ & - & - & - & & \\
\hline BAR & Buy & Call & 3.29 & $150,000.00$ & $493,500.00$ & EUR/PLN & $2008-7-22$ & $2009-1-21$ & $2009-2-6$ & 3.13 & Settled & fx settlement & 4.3305 & $156,075.00$ & $62,430.00$ & $156,075.00$ \\
\hline BAR & Buy & Call & 3.29 & $150,000.00$ & $493,500.00$ & EUR/PLN & $2008-7-22$ & $2009-2-4$ & $2009-2-20$ & 3.13 & $\begin{array}{l}\text { The option sold } \\
\text { back }\end{array}$ & - & - & - & & \\
\hline BAR & Buy & Call & 3.29 & $150,000.00$ & $493,500.00$ & EUR/PLN & $2008-7-22$ & $2009-2-18$ & $2009-3-6$ & 3.13 & $\begin{array}{l}\text { The option sold } \\
\text { back }\end{array}$ & - & - & - & & \\
\hline BAR & Buy & Call & 3.29 & $150,000.00$ & $493,500.00$ & EUR/PLN & $2008-7-22$ & 2009-3-4 & 2009-3-20 & 3.13 & Settled & fx settlement & 4.7432 & $217,980.00$ & $87,192.00$ & $217,980.00$ \\
\hline BAR & Buy & Call & 3.29 & $150,000.00$ & $493,500.00$ & EUR/PLN & $2008-7-22$ & 2009-3-18 & $2009-4-3$ & 3.13 & Settled & fx settlement & 4.5313 & $186,195.00$ & $74,478.00$ & $186,195.00$ \\
\hline BAR & Buy & Call & 3.29 & $150,000.00$ & $493,500.00$ & EUR/PLN & $2008-7-22$ & $2009-4-1$ & 2009-4-17 & 3.13 & Settled & fx settlement & 4.6462 & $203,430.00$ & $81,372.00$ & $203,430.00$ \\
\hline BAR & Buy & Call & 3.29 & $150,000.00$ & $493,500.00$ & EUR/PLN & 2008-7-22 & 2009-4-15 & $2009-5-4$ & 3.13 & Settled & fx settlement & 4.2351 & $141,765.00$ & $56,706.00$ & $141,765.00$ \\
\hline BAR & Buy & Call & 3.29 & $150,000.00$ & $493,500.00$ & EUR/PLN & $2008-7-22$ & 2009-4-29 & $2009-5-15$ & 3.13 & Settled & fx settlement & 4.4498 & $173,970.00$ & $69,588.00$ & $173,970.00$ \\
\hline BAR & Buy & Call & 3.29 & $150,000.00$ & $493,500.00$ & EUR/PLN & $2008-7-22$ & $2009-5-13$ & 2009-5-29 & 3.13 & Settled & fx settlement & 4.3943 & $165,645.00$ & $66,258.00$ & $165,645.00$ \\
\hline BAR & Buy & Call & 3.29 & $150,000.00$ & $493,500.00$ & EUR/PLN & $2008-7-22$ & $2009-5-27$ & 2009-6-15 & 3.13 & Settled & fx settlement & 4.4313 & $171,195.00$ & $68,478.00$ & $171,195.00$ \\
\hline BAR & Buy & Call & 3.29 & $150,000.00$ & $493,500.00$ & EUR/PLN & $2008-7-22$ & 2009-6-10 & $2008-7-25$ & 3.13 & $\begin{array}{l}\text { The option sold } \\
\text { back }\end{array}$ & - & - & 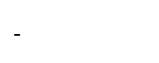 & & \\
\hline BAR & Buy & Call & 3.29 & $150,000.00$ & $493,500.00$ & EUR/PLN & $2008-7-22$ & $2009-6-24$ & $2008-8-8$ & 3.13 & $\begin{array}{l}\text { The option sold } \\
\text { back }\end{array}$ & - & - & - & & \\
\hline \multirow[t]{3}{*}{ BAR } & Buy & Call & 3.29 & $150,000.00$ & $493,500.00$ & EUR/PLN & $2008-7-22$ & $2009-7-8$ & 2008-8-22 & 3.13 & $\begin{array}{l}\text { The option sold } \\
\text { back }\end{array}$ & - & - & - & & \\
\hline & & & & & & & & & & & & & & $\underline{1,742,280.50}$ & $\underline{692,898.50}$ & $\underline{1,731,131.33}$ \\
\hline & & & & & & & & & & & & & & \multicolumn{3}{|c|}{ "-": the bank I pays; "+": the bank I receives } \\
\hline
\end{tabular}

Source: Own study. 


\section{Conclusions}

In the cases discussed, the option strategies concluded by companies with banks in Poland at the period 2007-2008 were mostly risk reversals, i.e., zero cost strategies involving buying puts by clients and selling call options. Call options nominal was even twice the put notional, as the puts built into the risk reversals were sold by banks in-the-money. The discrepancy between long puts and short calls nominal captured also banks' margin. Moreover, there were both European and American barriers attached to these options. These barriers were down and out or up and in. Should put options sold by banks get deeper ITM, structures would extinguish thus limiting banks' exposition. There were also other loss-stoppers installed: In case of TARNSs, it was a cap value of total redemption on an underlying option received by the corporate client that switched off the whole structure. Apparently, the underpriced OTM call options sold by companies had no payoff stopper in case the underlying currency (the euro) would harshly appreciate.

Exporters agreed to involve into such transaction experiencing persistent upsurge of Polish currency against euro since Poland's EU admission. The EUR/PLN exchange rate started to change its trend only before the Lehman collapse on 15th September, 2008. Zloty suddenly depreciated by over $20 \%$ together with the EUR/PLN implied volatility increasing six-fold.

Over-hedged with short call options, companies occurred tremendous losses cumulating in the year 2009. Implied volatility jump increased short calls premium triggering margin calls.

In view of those "toxic" currency options, the question was raised in Poland to what extent such transactions were "fair" operations. That was quite an important point in the pertinent legal issue, as heavy losses sustained on options had occasionally touched off legal disputes, with quite a few brought before courts. This paper tries to answer the question with the discussed transactions in mind. This paper assumes that there was no case of overhedge, i.e., the bank had accurately calculated client's currency position. It is also taken for granted that the bank acted loyally, i.e., that as a financial institution it could not possibly have foreseen the drastic change of trend on the currency market.

What can certainly be held against the bank is its imposition of a high broker margin on its client. Had the bank contained its appetite solely to the market spread of the time and had it offered the firm improved terms of the transaction contract, the amount of loss on the options would have been as much as by one-fourth smaller than posted. In that case, as in all other ones, the question of how to advise client appropriately on risks involved in the use of hedging instruments remains unanswered. In 2008, it will be recalled that sales units of banks were aggressively approaching businesses to offer them currency options. Poland at the time was late in implementing the MiFID directive ${ }^{1}$. The EU regulation bans the taking of advantage of expertise in concluding contracts involving financial instruments. The MiFID aims to harmonize investment operations and seeks to

1 The MiFID directive (markets in financial instruments directive) consists of three regulations:

(1) MiFID I: Directive 2004/39/EC of the European Parliament and of the Council of 21 April 2004 on markets in financial instruments, which sets out the scope of regulation in detail;

(2) MiFID II: Commission Directive 2006/73/EC of 10 August, 2006 implementing Directive 2004/39/EC of the European Parliament and of the Council as regards organizational requirements and operating conditions for investment firms and defined terms for the purposes of that Directive (implementing regulation);

(3) Commission Regulation (EC) No. 1287/2006 of 10 August, 2006 implementing Directive 2004/39/EC of the European Parliament and of the Council as regards record-keeping obligations for investment firms, transaction reporting, market transparency, admission of financial instruments to trading, and defined terms for the purposes of that Directive (implementing regulation). 
introduce uniform conduct of business rules in the provision of investment services in European Union countries, with protection of clients of investment firms the declared guiding idea behind the directive. Poland was obligated to implement the MiFID provisions in the relevant Polish regulations by 1 November, 2007. The Polish President first asked the Constitutional Tribunal to check the implementing act for compliance with Polish laws. It was only on 29 September, 2009 that the President signed the act into law.

\section{References}

Cloke, J. (2009). An economic wonderland: Derivative castle built on sand. Critical Perspectives on International Business, $5(1 / 2), 107-119$.

DeRosa, D. F. (2011). Options on foreign exchange (3rd ed.). Hoboken NJ, USA: Wiley Finance.

Glass, A. W. (2009). The regulatory drive towards central counterparty clearing of OTC credit derivatives and the necessary limits on this. Capital Markets Law Journal, 4, 79-98.

GUS. (2010). Instrumenty pochodne w portfelach przedsiębiorstw niefinansowych. Raport $\mathrm{z}$ badania (Derivative instruments in nonfinancial enterprise portfolios. Research report of Central Statistical Office of Poland). Warsaw: Główny Urząd Statystyczny.

Haug, E. G. (2007). The complete guide to option pricing formulas. New York: McGraw-Hill.

Konopczak, M., Mielus, P., \& Wieprzowski, P. (2011). Rynkowe aspekty problemów na pozagiełdowym rynku instrumentów pochodnych w Polsce w czasie globalnego kryzysu finansowego (Market aspects of the Polish OTC derivatives market problems during global financial crisis). Bank i Kredyt, 42(2), 97-124.

Kwong, T., Fok, D., Kwong, K. K., Fok, L., \& Fok, W. (2011). An explanatory study of a risk neutral pricing model for accumulators. Bingley, West Yorkshire: Emerald Group Published Limited.

Liberadzki, K. (2015). The issue of "toxic" foreign exchange options in Poland. Jordan Journal of Economic Sciences, $2(1), 87-95$.

Mullineux, A. W. (2010). Financial innovation and social welfare. Journal of Financial Regulation and Compliance, 18, $243-256$.

National Bank of Poland [NBP]. (2009). Raport o stabilności systemu finansowego czerwiec 2009 (Financial system stability June 2009 report). Warsaw: National Bank of Poland.

Polish Financial Supervision Authority [KNF]. (2008). Komunikat KNF z dnia 17 grudnia 2008 r. w sprawie wpływu walutowych instrumentów pochodnych na banki i spółki publiczne (Polish Financial Supervisory Office announcement: On the impact of currency derivative instruments on banks and public liability companies). Warsaw: Komisja Nadzoru Finansowego.

Polish Financial Supervision Authority [KNF]. (2009a). Podstawowe wnioski z analizy zaangażowania przedsiębiorstw w walutowe instrumenty pochodne (Polish Financial Supervision Authority report: Main findings of an analysis of enterprises' engagement in currency derivative instruments). Warsaw: Komisja Nadzoru Finansowego.

Polish Financial Supervision Authority [KNF]. (2009b). Aktualizacja danych o zaangażowaniu przedsiębiorstw w walutowe instrumenty pochodne (Polish Financial Supervision Authority report: Update on enterprises' engagement in currency derivative instruments). Warsaw: Komisja Nadzoru Finansowego.

Polish Financial Supervision Authority [KNF]. (2009c). Komunikat Urzędu KNF w sprawie zaangażowania przedsiębiorstw w walutowe instrumenty pochodne (Polish Financial Supervision Authority announcement: On enterprises' engagement in currency derivative instruments on banks and public liability companies). Warsaw: Komisja Nadzoru Finansowego.

Razin, A., \& Rosefielde, S. (2011). Currency and financial crises of the 1990s and 2000s. CESifo Economic Studies, 57, 499-530.

Sikorski, D. (2011). The global financial crisis. The Impact of the Global Financial Crisis on Emerging Markets, 93, 17-90. 